\title{
Nowhere-zero 3-flows in squares of graphs
}

\author{
Rui Xu and Cun-Quan Zhang* \\ Department of Mathematics \\ West Virginia University, West Virginia, USA \\ xu@math.wvu.edu, cqzhang@math.wvu.edu
}

Submitted: May 31, 2002; Accepted: Jan 15, 2003; Published: Jan 22, 2003

MR Subject Classifications: 05C15, 05C20, 05C70, 05C75, 90B10

\begin{abstract}
It was conjectured by Tutte that every 4-edge-connected graph admits a nowherezero 3-flow. In this paper, we give a complete characterization of graphs whose squares admit nowhere-zero 3-flows and thus confirm Tutte's 3-flow conjecture for the family of squares of graphs.
\end{abstract}

\section{Introduction}

All graphs considered in this paper are simple. Let $G=(V, E)$ be a graph with vertex set $V$ and edge set $E$. For any $v \in V(G)$, we use $d_{G}(v), N_{G}(v)$ to denote the degree and the neighbor set of $v$ in $G$, respectively. The minimal degree of a vertex of $G$ is denoted by $\delta(G)$. We use $K_{m}$ for a complete graph on $m$ vertices, $P_{t}$ for a path of length $t$ and $W_{4}$ for a graph obtained from a 4-circuit by adding a new vertex $x$ and edges joining $x$ to all the vertices on the circuit. We call $x$ the center of this $W_{4}$ and each edge with $x$ as one end is called a center edge. Let $D$ be an orientation of $G$. Then the set of all edges with tails (or heads) at a vertex $v$ is denoted by $E^{+}(v)$ (or $E^{-}(v)$ ). If an edge $u v$ is oriented from $u$ to $v$ under $D$, then we say $D(u v)=u \rightarrow v$. The square of $G$, denoted by $G^{2}$, is the graph obtained from $G$ by adding all the edges that join distance 2 vertices in $G$. We refer the reader to [1] for terminology not defined in this paper.

Definition 1.1 Let $D$ be an orientation of $G$ and $f$ be a function: $E(G) \mapsto Z$. Then

(1). The ordered pair $(D, f)$ is called a $k$-flow of $G$ if $-k+1 \leq f(e) \leq k-1$ for every edge $e \in E(G)$ and $\sum_{e \in E^{+}(v)} f(e)=\sum_{e \in E^{-}(v)} f(e)$ for every $v \in V(G)$.

(2). The ordered pair $(D, f)$ is called a Modular $k$-flow of $G$ if for every $v \in V(G)$, $\sum_{e \in E^{+}(v)} f(e) \equiv \sum_{e \in E^{-}(v)} f(e)(\bmod k)$.

${ }^{*}$ Partially supported by the National Security Agency under Grant MDA904-01-1-0022. 
The support of a $k$-flow (Modular $k$-flow) $(D, f)$ of $G$ is the set of edges of $G$ with $f(e) \neq 0(f(e) \not \equiv 0(\bmod k))$, and is denoted by $\operatorname{supp}(f)$. A $k$-flow $(D, f)$ (Modular $k$-flow) of $G$ is nowhere-zero if $\operatorname{supp}(f)=E(G)$.

For convenience, a nowhere-zero $k$-flow is abbreviated as a $k$-NZF. The concept of integer-flow was introduced by Tutte([7, 8] also see $[9,4])$ as a refinement and generalization of the face-coloring and edge-3-coloring problems. One of the most well known open problems in this subject is the following conjecture due to Tutte:

Conjecture 1.2 (Tutte, unsolved problem 48 in [1]) Every 4-edge-connected graph admits a $3-N Z F$.

Squares of graphs admitting 3-NZF's are to be characterized in this paper. The following families of graphs are the exceptions in the main theorem.

Definition $1.3 \mathcal{T}_{1,3}=\left\{T \mid T\right.$ is a tree and $d_{T}(v)=1$ or 3 for every $\left.v \in V(T)\right\}$

Definition 1.4 $\overline{\mathcal{T}}_{1,3}=\left\{T \mid T \in \mathcal{T}_{1,3}\right.$ or $T$ is a 4-circuit or $T$ can be obtained from some $T^{\prime} \in \mathcal{T}_{1,3}$ by adding some edges each of which joins a pair of distance 2 leaves of $\left.T^{\prime}\right\}$

The following is the main result of this paper.

Theorem 1.5 Let $G$ be a connected simple graph. Then $G^{2}$ admits a 3-NZF if and only if $G \notin \overline{\mathcal{T}}_{1,3}$.

An immediate corollary of Theorem 1.5 is the following partial result to Tutte's 3 -flow conjecture (Conjecture 1.2).

Corollary 1.6 Let $G$ be a graph. If $\delta\left(G^{2}\right) \geq 4$ then $G^{2}$ admits a 3-NZF.

This research is motivated by Conjecture 1.2 and the following open problem:

Conjecture 1.7 (Zhang [11]) If every edge of a 4-edge-connected graph $G$ is contained in a circuit of length at most 3 or 4 , then $G$ admits a 3-NZF.

Theorem 1.5 and the following early results are partial results of the open problem above.

Theorem 1.8 (Catlin [2]) If every edge of a graph $G$ is contained in a circuit of length at most 4 , then $G$ admits a $4-N Z F$.

Theorem 1.9 (Lai [5]) Every 2-edge-connected, locally 3-edge-connected graph admits a $3-N Z F$.

Theorem 1.10 (Imrich and Skrekovski [3]) Let $G$ and $H$ be two graphs. Then $G \times H$ admits a $3-N Z F$ if both $G$ and $H$ are bipartite. 


\section{Splitting operation, flow extension and lemmas}

Definition 2.1 (A special splitting operation) Let $G$ be a graph and $e=x y \in E(G)$. The graph $G_{* e}$ is obtained from $G$ by deleting the edge $e$ and adding two new vertices $x^{\prime}$ and $y^{\prime}$ and adding two new edges, $e_{x}$ and $e_{y}$, joining $x$ and $y^{\prime}, y$ and $x^{\prime}$, respectively.

Definition 2.2 Let $G$ be a graph, let $(D, f)$ be a 3-flow of $G$ and let $F \subseteq E(G) \backslash$ supp $(f)$. $A$ 3-flow $\left(D^{\prime}, f^{\prime}\right)$ of $G$ is called an $(F, f)$-changer if $F \cup \operatorname{supp}(f) \subseteq \operatorname{supp}\left(f^{\prime}\right)$.

Lemma 2.3 ([7]) A graph $G$ admits a $k$-flow $\left(D, f_{1}\right)$ if and only if $G$ admits a Modular $k$-flow $\left(D, f_{2}\right)$ such that $f_{1}(e) \equiv f_{2}(e)(\bmod k)$ for each $e \in E(G)$.

An orientation of a graph $G$ is called a modular 3-orientation if $\left|E^{+}(v)\right| \equiv\left|E^{-}(v)\right|(\bmod 3)$, for every $v \in V(G)$. The following result appears in $[4,6,9]$, but by Lemma 2.3, we can attribute it to Tutte.

Lemma 2.4 ([7]) Let $G$ be a graph. Then $G$ admits a 3-NZF if and only if $G$ has a modular 3-orientation.

A partial 3-orientation $D$ of $G$ is an orientation of some edges of $G$ satisfying $\left|E^{+}(v)\right| \equiv\left|E^{-}(v)\right|(\bmod 3)$, for any $v \in V(G)$. The support of $D$ is the set of edges oriented under $D$ and is denoted by $\operatorname{supp}(D)$. Clearly the partial orientation obtained by reversing every oriented edge of a partial 3-orientation is also a partial 3-orientation.

Let $D$ be a partial 3-orientation of $G$ and let $C=v_{0} v_{1} \cdots v_{k-1} v_{0}$ be a circuit of $G$. A circuit-operation along $C$ is defined as following: For $0 \leq i \leq k-1$, if $D\left(v_{i} v_{i+1}\right)=v_{i} \rightarrow$ $v_{i+1}(\bmod k)$, then reverse the direction of this edge; if $\left(v_{i} v_{i+1}\right)(\bmod k)$ is not oriented under $D$, then orient it as $v_{i} \rightarrow v_{i+1}$; if $D\left(v_{i} v_{i+1}\right)=v_{i+1} \rightarrow v_{i}(\bmod k)$ then $v_{i} v_{i+1}$ loses it's orientation.

Lemma 2.5 Let $G$ be a graph, $(D, f)$ be a 3-flow of $G$ and $H$ be a subgraph of $G$

(1). If $H \cong W_{4}$ and $e \in E(H) \backslash \operatorname{supp}(f)$ is a center edge, then an $(\{e\}, f)$-changer exists.

(2). If $H$ is a circuit of length 3 with $E(H) \cap \operatorname{supp}(f)=\{e\}$, then an $(E(H) \backslash\{e\}, f)$ changer exists.

Proof. (1). Since $H \cong W_{4}$, let $x$ be the center of $H$ and let $u_{1} u_{2} u_{3} u_{4} u_{1}$ be the 4circuit $H \backslash x$. Since $G$ has a 3 -flow $(D, f)$, then $G$ has a partial 3-orientation $D^{*}$ with $\operatorname{supp}\left(D^{*}\right)=\operatorname{supp}(f)$. We need only to find a partial 3-orientation $D^{\prime}$ such that $\operatorname{supp}\left(D^{*}\right) \cup$ $\{e\} \subseteq \operatorname{supp}\left(D^{\prime}\right)$. Since $e$ is a center edge, without loss of generality, assume that $e=x u_{1}$.

First we assume $E(H) \backslash\{e\} \subseteq \operatorname{supp}\left(D^{*}\right)$. Without loss of generality, assume $D^{*}\left(u_{1} u_{2}\right)=$ $u_{1} \rightarrow u_{2}$. Then $D^{*}\left(u_{2} x\right)=x \rightarrow u_{2}$. Otherwise, we do a circuit-operation along $u_{1} u_{2} x u_{1}$ and then get a needed partial 3-orientation $D^{\prime}$ of $G$. For the same reason, $u_{4}$ must be the tail (or head) of both $u_{1} u_{4}$ and $x u_{4}$. By symmetry, we consider the following two cases.

Case 1. $D^{*}\left(u_{1} u_{4}\right)=u_{1} \rightarrow u_{4}$ and $D^{*}\left(x u_{4}\right)=x \rightarrow u_{4}$. 
We may assume that $u_{3}$ is the tail (or head) of all edges incident with it in $H$. Otherwise, there exists a directed 2-path $x u_{3} u_{i}$ (or $u_{i} u_{3} x$ ) for some $i \in\{2,4\}$. Then we do circuit-operations along $x u_{3} u_{i} x$ (or $u_{i} u_{3} x u_{i}$ ) and along $u_{1} u_{i} x u_{1}$. Therefore, we get a needed partial 3-orientation of $D^{\prime}$ of $G$.

If all edges in $H$ have $u_{3}$ as a tail, then we do circuit-operations along $x u_{1} u_{4} x$, along $u_{4} x u_{3} u_{4}$, along $x u_{3} u_{2} x$ and along $u_{2} x u_{1} u_{2}$; If all edges in $H$ have $u_{3}$ as a head, then we do circuit-operations along $u_{1} u_{2} u_{3} x u_{1}$ and along $u_{3} x u_{4} u_{3}$. In both cases, we get a needed partial 3-orientation $D^{\prime}$ of $G$.

Case 2. $D^{*}\left(u_{1} u_{4}\right)=u_{4} \rightarrow u_{1}$ and $D^{*}\left(x u_{4}\right)=u_{4} \rightarrow x$.

Similar to Case 1 , we may assume $u_{3}$ be the tail (or head) of all edges incident with it in $H$. If all edges in $H$ have $u_{3}$ as a tail, then we do circuit-operations along $x u_{1} u_{4} x$, along $u_{3} u_{4} u_{1} u_{2} u_{3}$ and along $u_{3} x u_{2} u_{3}$; If all edges in $H$ have $u_{3}$ as a head, then we do circuit-operations along $u_{1} x u_{2} u_{1}$, along $u_{4} u_{1} u_{2} u_{3} u_{4}$ and along $u_{4} x u_{3} u_{4}$. In both cases, we get a needed partial 3-orientation $D^{\prime}$ of $G$.

If $\operatorname{supp}\left(D^{*}\right)$ misses some other edges of $E(H)$, say $e^{*}=a b \in E(H) \backslash \operatorname{supp}\left(D^{*}\right)$, then we define $D^{*}(a b)=a \rightarrow b$ or $b \rightarrow a$, by the proof of Case 1 and Case 2, we can find a needed $D^{\prime}$ of $G$.

(2). it is trivial.

Lemma 2.6 For each $G \in \overline{\mathcal{T}}_{1,3}$ and each $e_{0} \in E(G)$, the graph $G^{2}$ admits a 3-flow $(D, f)$ such that $\operatorname{supp}(f)=E\left(G^{2}\right) \backslash\left\{e_{0}\right\}$

Proof. Induction on $|E(G)|$. It is obviously true for graphs $G$ with $G^{2}=K_{4}$ (including $G=C_{4}$, the circuit of length 4). So, assume that $|V(G)| \geq 5$ and let $D$ be any fixed orientation of $G^{2}$.

Let $e=x y$ with $d_{G}(x)=d_{G}(y)=3$. Then $G_{* e}$ consists of two components, say $G_{1}$ and $G_{2}$. Clearly, $G_{1}, G_{2} \in \overline{\mathcal{T}}_{1,3}$. Without loss of generality, let $e_{0} \in E\left(G_{1}\right)$. By induction, $G_{1}^{2}$ admits a 3 -flow $\left(D, f_{1}\right)$ such that $\operatorname{supp}\left(f_{1}\right)=E\left(G_{1}^{2}\right) \backslash\left\{e_{0}\right\}$ and $G_{2}^{2}$ admits a 3-flow $\left(D, f_{2}\right)$ that $\operatorname{supp}\left(f_{2}\right)=E\left(G_{2}^{2}\right) \backslash\{e\}$.

Then, identifying the split vertices and edges, back to $G,\left(D, f_{1}+f_{2}\right)$ is a 3 -flow $(D, f)$ with $\operatorname{supp}(f)=E\left(G^{2}\right) \backslash\left\{e_{0}\right\}$.

Lemma 2.7 (1). Let $G$ be a $k$-path with $k \geq 2$ or an $m$-circuit with $m=3$ or $m \geq 5$. Then $G^{2}$ admits a $3-N Z F$.

(2). Let $G$ be a graph obtained from an $r$-circuit $x_{0} x_{1} \cdots x_{r-1} x_{0}$ by attaching an edge $x_{i} v_{i}$ at each $x_{i}$ for $0 \leq i \leq r-1$, where $v_{i} \neq v_{j}$ if $i \neq j$. Then $G^{2}$ admits a 3-NZF.

(3). Let $G$ be a graph obtained from an $m$-circuit $x_{0} x_{1} \cdots x_{m-1} x_{0}$ by attaching an edge $x_{m-1} v$ at $x_{m-1}$ alone, where $m \geq 5$. Then $G^{2}$ admits a $3-N Z F$.

Proof. (1). If $G$ is an $m$-circuit with $m=3$ or $m \geq 5$, then $G^{2}$ is a cycle (every vertex is of even degree) and $G^{2}$ admits 2 -NZF. If $G$ is a $k$-path with $k \geq 2$, by induction on $k$ and using Lemma 2.5-(2), $G^{2}$ admits a $3-\mathrm{NZF}$.

(2). For $r \geq 5$ (or $r=3$ ): let $D$ be an orientation such that $v_{i}(0 \leq i \leq r-1)$ is the tail of every edge of $G^{2}$ incident with it and all the other edges are oriented as 
$x_{i} \rightarrow x_{i+1}, x_{i} \rightarrow x_{i+2}(\bmod r)\left(\right.$ or $x_{i} \rightarrow x_{i+1}(\bmod 3)$ only for $\left.r=3\right)$. Obviously, $D$ is a modular 3-orientation of $G^{2}$.

For $r=4$ : let $D$ be the orientation such that $v_{0}$ and $v_{2}$ be the tail of every edge of $G^{2}$ incident with it, $v_{1}$ and $v_{3}$ be the head of every edge of $G^{2}$ incident with it, $x_{0} x_{1} x_{3} x_{2} x_{0}$ as a directed circuit and other edges are oriented as $x_{3} \rightarrow x_{0}, x_{1} \rightarrow x_{2}$. Obviously, $D$ is a modular 3-orientation of $G^{2}$.

(3). Orient all the edges as $x_{i} \rightarrow x_{i+1}, x_{i} \rightarrow x_{i+2}(\bmod m)$ for $0 \leq i \leq m-1$ and let $v$ be the tail of every edge of $G^{2}$ incident with it. Then reverse the direction of the following edges: $x_{0} x_{m-1}, x_{0} x_{m-2}$. Clearly, this orientation is a modular 3-orientation of $G^{2}$.

\section{Proof of the main theorem}

Proof. $\Longrightarrow$ By contradiction. Suppose $G \in \overline{\mathcal{T}}_{1,3}$. Let $G$ be a counterexample with $|V(G)|+|E(G)|$ as small as possible. Clearly $|V(G)| \geq 5$ and $G$ contains no circuits. So $G \in \mathcal{T}_{1,3}$. Let $v \in V(G)$ be a degree 3 vertex such that $N_{G}(v)=\left\{v_{1}, v_{2}, v_{3}\right\}, d_{G}\left(v_{1}\right)=$ $d_{G}\left(v_{2}\right)=1$. Clearly, $G_{1}=G \backslash\left\{v_{1}, v_{2}\right\} \in \mathcal{T}_{1,3}$. Since $G^{2}$ has a modular 3-orientation $D$ and both $v_{1}$ and $v_{2}$ are degree 3 vertices in $G^{2}$, then this orientation restricted to the edge set of $G_{1}^{2}$ will generate a modular 3-orientation of $G_{1}^{2}$. Therefore, $G_{1}^{2}$ admits a 3-NZF, a contradiction.

$\Longleftarrow$ Let $G$ be a counterexample to the theorem such that

(i). $|E(G)|-|V(G)|$ is as small as possible,

(ii). subject to (i), $|E(G)|$ is as small as possible.

Note that $|E(G)|-|V(G)|+1$ is the rank of the cycle space of $G$.

Claim 1. Let $e_{0}=x y \in E(G)$. If $d_{G}(x) \geq 3$ and $d_{G}(y) \geq 2$, then $x y$ is not a cut edge of $G$.

If $e_{0}$ is a cut-edge, then at least one component of $G_{* e_{0}}$ is not in $\overline{\mathcal{T}}_{1,3}$, say, $G_{1}$ is not, while $G_{2}$ might be. By induction, let $\left(D, f_{i}\right)$ be a 3 -flow of $G_{i}^{2}$ for each $i=1,2$ such that $f_{1}$ is nowhere-zero, $f_{2}$ might miss only one edge $e_{x}$ (that is a copy of $e_{0}$ ). Without loss of generality, assume that $f_{1}\left(e_{y}\right)+f_{2}\left(e_{x}\right) \not \equiv 0(\bmod (3))$. Then, identifying the split vertices and edges, back to $G,\left(D, f_{1}+f_{2}\right)$ is a nowhere-zero Modular 3-flow of $G^{2}$. By Lemma 2.3, $G^{2}$ admits a $3-\mathrm{NZF}$, a contradiction.

Claim 2. $d_{G}(x) \leq 3$ for any $x \in V(G)$.

Otherwise, assume that $d_{G}(x) \geq 4$ for some vertex $x \in V(G)$. Clearly $G \approx K_{1, m}$ for $m \geq 4$ since $K_{1, m}$ is not a counterexample. So there exists $e_{0}=x y \in E(G)$ with $d_{G}(y) \geq 2$. By Claim $1, e_{0}$ is not a cut edge of $G$ and $G_{1}=G_{* e_{0}} \notin \overline{\mathcal{T}}_{1,3}$. Then by (i), $G_{1}^{2}$ admits a $3-\mathrm{NZF}$.

In $G_{1}^{2}$, identify $x$ and $x^{\prime}, y$ and $y^{\prime}$, and use one edge to replace two parallel edges, by Lemma 2.3, we will get $G^{2}$ and a Modular 3-flow $(D, f)$ of $G^{2}$ such that $E\left(G^{2}\right) \backslash \operatorname{supp}(f) \subseteq$ $\left\{x v\right.$ or $\left.y w \mid v \in N_{G}(y), w \in N_{G}(x)\right\}$. Let $C(x)=G^{2}\left[N_{G}(x) \cup\{x\}\right]$. Then $C(x)$ is a clique of order at least 5 . We are to adjust $(D, f)$ so that the resulting Modular 3 -flow $\left(D, f^{\prime}\right)$ 
of $G^{2}$ misses only edges of $\{u v \mid u, v \in V(C(x))\}$. For each edge $x v$ which is missed by $\operatorname{supp}(f)$ and $x v \notin E(C(x))$, xyvx must be a circuit of $G^{2}$, so let $\left(D, f_{x v}\right)$ be a 3-flow of $G^{2}$ with $\operatorname{supp}\left(f_{x v}\right)=\{x y, y v, x v\}$ and $f_{x v}(y v)+f(y v) \not \equiv 0(\bmod 3)$. Now $\left(D, f+f_{x v}\right)$ is a Modular 3-flow of $G^{2}$ whose support contains $x v, y v$, but may miss $x y$. Repeat this adjustment and do the similar adjustment for the edges $y w$ not in the support until we get a Modular 3-flow $\left(D, f^{\prime}\right)$ of $G^{2}$ such that $E\left(G^{2}\right) \backslash \operatorname{supp}\left(f^{\prime}\right) \subseteq E(C(x))$. Since each edge in $C(x)$ is contained in some $K_{5}$ and thus is a center edge in some $W_{4}$, by Lemma 2.3 and Lemma 2.5-(1), $G^{2}$ admits a 3-NZF, a contradiction.

Claim 3. No degree 2 vertex is contained in a 3-circuit.

By contradiction. Assume $x y z x$ is a circuit of $G$ with $d_{G}(x)=2$. If $d_{G}(y)=2$, then we must have $d_{G}(z)=3$. Therefore $G_{1}=G \backslash\{x y\} \notin \overline{\mathcal{T}}_{1,3}$ and $G_{1}^{2}=G^{2}$, contradicting (ii). So $d_{G}(y)=d_{G}(z)=3$.

Let $N_{G}(y)=\left\{x, y^{\prime}, z\right\}$ and $N_{G}(z)=\left\{x, y, z^{\prime}\right\}$. Let $G_{1}=G-\{x\}$. Since $\left(N_{G}(y) \cap\right.$ $\left.N_{G}(z)\right) \backslash\{x\}=\emptyset$ (otherwise, let $G_{2}=G \backslash\{y z\}$, then $G_{2}^{2}=G^{2}, G_{2} \notin \overline{\mathcal{T}}_{1,3}$, contradicting (ii)) and $d_{G_{1}}(y)=2$, then $G_{1} \notin \overline{\mathcal{T}}_{1,3}$. So $G_{1}^{2}$ admits a 3 -NZF. Since $E\left(G^{2}\right) \backslash E\left(G_{1}^{2}\right)=$ $\left\{x y, x y^{\prime}, x z, x z^{\prime}\right\}$, by Lemma $2.5-(2), G^{2}$ admits a $3-\mathrm{NZF}$, a contradiction.

Claim 4. No degree 2 vertex of $G$ is contained in a 4-circuit.

Assume $C=x u_{1} u_{2} u_{3} x$ is a 4-circuit of $G$ and $d_{G}(x)=2$. By Claim 3, $u_{1} u_{3} \notin E(G)$. Let $u_{i}^{\prime}$ be the adjacent vertex of $u_{i}$ which is not in $V(C)$ if $d_{G}\left(u_{i}\right)=3$ for some $i \in\{1,2,3\}$. Let $G_{1}=G \backslash\{x\}$. We consider the following 3 cases.

Case 1. $d_{G}\left(u_{1}\right)=d_{G}\left(u_{3}\right)=2$.

Then $d_{G}\left(u_{2}\right)=3$ and $d_{G}\left(u_{2}^{\prime}\right) \geq 2$ (if $d_{G}\left(u_{2}^{\prime}\right)=1$, it's easy to show $G^{2}$ admits a 3-NZF). Clearly, $u_{2} u_{2}^{\prime}$ is a cut edge, contradicting Claim 1.

Case 2. Exactly one of $u_{1}, u_{3}$ has degree 3.

Assume $d_{G}\left(u_{1}\right)=3$ and $d_{G}\left(u_{3}\right)=2$. Since $d_{G_{1}}\left(u_{1}\right)=2$, if $d_{G_{1}}\left(u_{1}^{\prime}\right)=2$ then $u_{1}^{\prime}$ is not contained in a 3 -circuit in $G$ (by Claim 3), and so $G_{1} \notin \overline{\mathcal{T}}_{1,3}$. By induction, $G_{1}^{2}$ admits a 3-NZF. Since $E\left(G^{2}\right) \backslash E\left(G_{1}^{2}\right)=\left\{x u_{1}^{\prime}, x u_{1}, x u_{2}, x u_{3}\right\}$ and $G^{2}\left[V(C) \cup\left\{u_{1}^{\prime}\right\}\right]$ contains a $W_{4}$ with $x$ as its center, by Lemma 2.5-(1), $G^{2}$ admits a $3-\mathrm{NZF}$, a contradiction.

Case 3. $d_{G}\left(u_{1}\right)=d_{G}\left(u_{3}\right)=3$.

If $u_{1}^{\prime}=u_{3}^{\prime}$, then $u_{1}^{\prime} u_{1} u_{2} u_{3}$ is a 3 -path, otherwise $u_{1}^{\prime} u_{1} u_{2} u_{3} u_{3}^{\prime}$ is 4 -path. In both cases $G_{1}^{2}$ admits a 3 -NZF. Since $E\left(G^{2}\right) \backslash E\left(G_{1}^{2}\right)=\left\{x u_{1}^{\prime}, x u_{1}, x u_{2}, x u_{3}, x u_{3}^{\prime}\right\}$ and each edge $x u_{i}$ or $x u_{j}^{\prime}$ is contained in some $W_{4}$ in $G^{2}$ as a center edge for $1 \leq i \leq 3$ and $j=1,3$, by Lemma 2.5-(1), $G^{2}$ admits a $3-\mathrm{NZF}$. a contradiction.

Claim 5. For any $v \in V(G), d_{G}(v) \neq 2$.

Otherwise, if there exists $v \in V(G)$ such that $d_{G}(v)=2$, then by Claim 3-4, $v$ is not contained in any circuits of length 3 or 4 . By Lemma 2.7-(1), $G$ cannot be a $k$-path with $k \geq 2$ or an $m$-circuit with $m=3$ or $m \geq 5$. Let us consider the following cases.

Case 1. There exists a path $P_{m}=v_{1} v_{2} \cdots v_{m}$ such that $m \geq 3, v=v_{t}$ for some $2 \leq t \leq m-1, d_{G}\left(v_{k}\right)=2$ for $2 \leq k \leq m-1$ and $d_{G}\left(v_{1}\right) \neq 2, d_{G}\left(v_{m}\right) \neq 2$.

Clearly, at least one of $v_{1}, v_{m}$ has degree 3 . If $d_{G}\left(v_{i}\right)=3$ for $i=1$, or $m$, let $N_{G}\left(v_{i}\right) \backslash V\left(P_{m}\right)=\left\{v_{i}^{\prime}, v_{i}^{\prime \prime}\right\}$. Clearly, $G_{1}=G \backslash\left\{v_{2}, v_{3}, \ldots, v_{m-1}\right\} \notin \overline{\mathcal{T}}_{1,3}$ (because by 
Claim 3, degree 2 vertices are not contained in any 3-circuits of $G$ ). By Claim $1, G_{1}$ is connected. So $G_{1}^{2}$ admits a $3-\mathrm{NZF}\left(D, f_{1}\right)$. By Lemma 2.7-(1), $P_{m}^{2}$ admits a 3-NZF $\left(D, f_{2}\right)$. Then $G^{2}$ admits a 3-flow $(D, f)$ with $\operatorname{supp}(f)=\operatorname{supp}\left(f_{1}\right) \cup \operatorname{supp}\left(f_{2}\right)$. By Claim 3-4, E $\left(G^{2}\right) \backslash \operatorname{supp}(f)=\left\{v_{2} v_{1}^{\prime}, v_{2} v_{1}^{\prime \prime}, v_{m-1} v_{m}^{\prime}, v_{m-1} v_{m}^{\prime \prime}\right\}$, then by Lemma 2.5-(2), $G^{2}$ admits a 3 -NZF, a contradiction.

Case 2. There exists a $m$-circuit $C=v_{1} v_{2} \cdots v_{m} v_{1}$ with $m \geq 5, d_{G}\left(v_{i}\right)=2$ for $1 \leq i \leq m-1, d_{G}\left(v_{m}\right)=3$ and $v=v_{t}$ for some $1 \leq t \leq m-1$.

Suppose that $v_{0} \in N_{G}\left(v_{m}\right) \backslash V(C)$. By Claim $1, d_{G}\left(v_{0}\right)=1$. So by Lemma 2.7-(3), $G^{2}$ admits a $3-\mathrm{NZF}$, a contradiction.

Claim 6. Let $e=x y \in E(G)$ with $d_{G}(x)=d_{G}(y)=3$. Then e is contained in a circuit of length 3 or 4.

By contradiction. Let $G_{1}$ be the graph obtained from $G$ by deleting the edge $e$ and adding a new vertex $y^{\prime}$ and a new edge $x y^{\prime}$. Since $G$ contains no degree 2 vertices and $d_{G_{1}}(y)=2$, then $G_{1} \notin \overline{\mathcal{T}}_{1,3}$. By Claim $1, e$ is not a cut edge of $G$, then by (i), $G_{1}^{2}$ admits a 3 -NZF $\left(D, f_{1}\right)$. Identify $y$ and $y^{\prime}$, the resulting 3 -flow $\left(D, f_{2}\right)$ in $G^{2}$ misses only two edges $y_{1} x$ and $y_{2} x$ where $N(y)=\left\{y_{1}, y_{2}, x\right\}$ (since $x y$ is not contained a circuit of length 3 or 4). By Lemma 2.5-(2), $G^{2}$ admits a $3-\mathrm{NZF}$, a contradiction.

Claim 7. For each $x \in V(G)$ with $d_{G}(x)=3,\left|N_{G}(x) \cap V_{3}\right| \leq 2$, where $V_{3}$ is the set of all the degree 3 vertices of $G$.

By contradiction. Assume that $U=\left\{u_{1}, u_{2}, u_{3}\right\}=N_{G}(x) \cap V_{3}$. Let $G_{1}=G \backslash\{x\}$. By Claim $1, G_{1}$ is connected. Since $G$ contains no degree 2 vertices, $G_{1} \notin \overline{\mathcal{T}}_{1,3}$ and $G_{1}^{2}$ admits a 3 -NZF $(D, f)$. By Claim 6 , each $x u_{i}(1 \leq i \leq 3)$ is contained a circuit of length at most 4. We consider the following 3 cases.

Case 1. $G[U]$ contains at least 2 edges.

Suppose that $u_{1} u_{2}, u_{2} u_{3} \in E(G)$. Let $u_{i}^{\prime} \in N_{G}\left(u_{i}\right) \backslash U$ for $i=1,3$. If $u_{1}^{\prime}=u_{3}^{\prime}$, then $G^{2}\left[U \cup\left\{u_{1}^{\prime}, x\right\}\right] \cong K_{5}$, by Lemma 2.5 -(1), we can get a 3 -NZF of $G^{2}$, a contradiction. If $u_{1}^{\prime} \neq u_{3}^{\prime}$, then $G\left[u_{1}^{\prime} u_{1} u_{2} u_{3} u_{3}^{\prime}\right]$ is a 4-path, by Lemma 2.5-(1) (similar to Case 3 of Claim $4)$, we can get a $3-\mathrm{NZF}$ of $G^{2}$, a contradiction.

Case 2. $G[U]$ contains exactly 1 edge.

Assume that $u_{1} u_{2} \in E(G)$. By Claim 6, each edge $x u_{i}(i=1,2,3)$ is contained in a circuit of length 3 or 4 . So we may assume $z \in\left(N_{G}\left(u_{2}\right) \cap N_{G}\left(u_{3}\right)\right) \backslash\{x\}$. Clearly, $G^{*}=G^{2}[U \cup\{x, z\}] \cong K_{5}$. Let $u_{i}^{\prime} \in N_{G}\left(u_{i}\right) \backslash(U \cup\{z\})$ for $i=1$, 3. Clearly, $E\left(G^{2}\right) \backslash$ $\operatorname{supp}(f) \subseteq E\left(G^{*}\right) \cup\left\{x u_{1}^{\prime}, x u_{3}^{\prime}\right\}$. Since $x u_{j} u_{j}^{\prime} x(j=1,3)$ is a circuit of $G^{2}$, we can get a 3-flow $\left(D, f_{1}\right)$ such that $E\left(G^{2}\right) \backslash \operatorname{supp}\left(f_{1}\right) \subseteq E\left(G^{*}\right)$. By Lemma 2.5-(1), we can get a 3-NZF of $G^{2}$, a contradiction.

Case 3. $G[U]$ contains no edges.

Assume that $z_{1} \in\left(N_{G}\left(u_{1}\right) \cap N_{G}\left(u_{2}\right)\right) \backslash\{x\}$ and $z_{2} \in\left(N_{G}\left(u_{1}\right) \cap N_{G}\left(u_{3}\right)\right) \backslash\{x\}$. Let $G_{2}=G \backslash\left\{x u_{1}\right\}$, then $G_{2} \notin \overline{\mathcal{T}}_{1,3}$ and $G_{2}^{2}$ admits a 3 -NZF $\left(D, f_{1}\right)$. Clearly, $E\left(G^{2}\right) \backslash$ $\operatorname{supp}\left(f_{1}\right)=\left\{x u_{1}\right\}$. Since $x u_{1}$ is contained in a $W_{4}$ which is contained in the graph induced by $\left\{u_{1}, z_{1}, u_{2}, u_{3}, x\right\}$ in $G^{2}$ with $x$ as center, by Lemma $2.5-(1)$, we can get a 3 -NZF of $G^{2}$, a contradiction. 
Final Step. By Claim 2, Claim 5 and Claim 7, all vertices of $G$ have degree 1 or 3 and each degree 3 vertex is adjacent to at most 2 degree 3 vertices. So $G\left[V_{3}\right]$ is a path or a circuit, hence $G$ must be a graph obtained from an $r$-circuit $x_{0} x_{1} \cdots x_{r-1} x_{0}$ by attaching an edge $x_{i} v_{i}$ at each $x_{i}$ for $0 \leq i \leq r-1$, where $v_{i} \neq v_{j}$ if $i \neq j$, or a path $x_{0} x_{1} \cdots x_{p}$ by attaching an edge $v_{i} x_{i}(1 \leq i \leq p-1)$ at each $x_{i}$, where $v_{i} \neq v_{j}$ if $i \neq j$. Clearly the latter case is a graph in $\overline{\mathcal{T}}_{1,3}$. By Lemma $2.7-(2), G^{2}$ admits a $3-\mathrm{NZF}$, a contradiction.

\section{References}

[1] J. A. Bondy and U. S. R. Murty, Graph Theory with Applications. Macmillan, London, (1976).

[2] P. A. Catlin, Double cycle covers and the Petersen graph, J. Graph Theory, 13 (1989) 465-483.

[3] W. Imrich and R. Skrekovski, A theorem on integer flows on Cartesian product of graphs, J. Graph Theory, (to appear).

[4] F. Jaeger, Nowhere-zero flow problems, in: L. Beineke and R. Wilson, eds., Selected Topics in Graph Theory 3 (Wiley, New York, 1988)71-95.

[5] H.-J. Lai. Nowhere-zero 3-flows in locally connected graphs, J. Graph Theory, (to appear).

[6] R. Steinberg and D. H. Younger, Grötzsch's theorem for the projective plane, Ars Combin., 28, (1989)15-31.

[7] W. T. Tutte, On the embedding of linear graphs in surfaces, Proc. London Math. Soc., Ser. 2, 51 (1949)474-483.

[8] W. T. Tutte, A contribution on the theory of chromatic polynomial, Canad. J. Math., 6 (1954)80-91.

[9] D. H. Younger, Interger flows, J. Graph Theory, 7 (1983)349-357.

[10] C. Q. Zhang, Integer Flows and Cycle Covers of Graphs, Marcel Dekker, New York (1997).

[11] C. Q. Zhang, Integer Flows and Cycle Covers, Plenary lecture at Graph Theory Workshop, Nanjing Normal University, April, 1998. 